Cahiers $d u$ MONDE RUSSE

\section{Cahiers du monde russe}

Russie - Empire russe - Union soviétique et États indépendants

$58 / 4 \mid 2017$

Varia

\title{
Slava Gerovitch, Soviet Space Mythologies: Public Images, Private Memories \& the Making of a Cultural Identity
}

\section{Gregory Dufaud}

\section{(2) OpenEdition}

12 Journals

\section{Édition électronique}

URL : http://journals.openedition.org/monderusse/10186

DOI : ERREUR PDO dans /localdata/www-bin/Core/Core/Db/Db.class.php L.34 : SQLSTATE[HYO00]

[2006] MySQL server has gone away

ISSN : $1777-5388$

\section{Éditeur}

Éditions de l'EHESS

\section{Édition imprimée}

Date de publication : 1 octobre 2017

Pagination : 742-744

ISBN : 978-2-7132-2698-4

ISSN : $1252-6576$

\section{Référence électronique}

Gregory Dufaud, «Slava Gerovitch, Soviet Space Mythologies: Public Images, Private Memories \& the Making of a Cultural Identity », Cahiers du monde russe [En ligne], 58/4 | 2017, mis en ligne le 01 octobre 2017, consulté le 05 janvier 2021. URL : http://journals.openedition.org/monderusse/10186 ; DOI : https://doi.org/ERREUR PDO dans /localdata/www-bin/Core/Core/Db/Db.class.php L.34: SQLSTATE[HYO00] [2006] MySQL server has gone away 
Slava GEROVITCH

\section{Soviet Space Mythologies \\ Public Images, Private Memories \& the Making of a Cultural Identity}

Pittsburgh : University of Pittsburgh Press, 2015, 232 p.

Slava Gerovitch a écrit un livre important où la clarté de l'analyse se conjugue à un récit souvent fort et poignant. L'ouvrage est constitué d'articles déjà publiés dans des revues, mais remaniés afin de former un tout cohérent. Il ne faudrait pas se laisser tromper par son titre, quelque peu restrictif. Car, si des chapitres portent bien sur la mythologie spatiale, le propos est plus général : à travers des éclairages toujours très documentés (archives, imprimés et entretiens), il présente une étude ample de la conquête spatiale, au croisement de l'histoire culturelle et de l'histoire des sciences, de l'histoire sociale et de l'histoire politique. Se plaçant au plus près des individus, S. Gerovitch met au jour la complexité des enjeux et donne à comprendre les évolutions du programme spatial dans ses continuités et ses ruptures. Il refuse en effet toute approche surplombante et son corollaire, la conviction de l'historien d'être plus malin que les acteurs de l'époque. De ce point de vue, c'est une remarquable leçon de méthode administrée par S. Gerovitch, avec finesse et sans jamais chercher à en imposer. Son histoire non seulement déconstruit la mythologie de l'espace, mais propose aussi une vision renouvelée et, pourrait-on ajouter apaisée, du passé soviétique. Il montre ainsi les raisons d'agir des protagonistes, dans le prolongement des avancées de l'historiographie, mais loin des analyses dualistes et caricaturales. D'où un certain étonnement de voir mobilisée au détour d'une page une citation de Juliane Fürst qui réduit le comportement des individus au cynisme et à l'intérêt instrumental (p. 34). Afin de lever les ambiguités, cette question des modalités et des motifs de l'action aurait sans doute mérité d'être explicitement thématisée et davantage discutée.

Ce sont quatre fils qui traversent l'ouvrage. Le premier tient à la capacité des acteurs à faire émerger des dispositifs $a d$ hoc. S. Gerovitch révèle le travail réalisé par Sergei Korolev pour se rallier des soutiens en différentes sphères, jusqu'au plus haut sommet du pouvoir. Il a ainsi pu mettre en place une structure informelle qui, regroupant des personnes issues d'administrations différentes, s'est affranchie des hiérarchies institutionnelles : le Conseil des concepteurs en chef (Sovet glavnyh konstruktorov) lui a alors permis de dépasser les pesanteurs administratives et de disposer de l'ensemble des moyens nécessaires à la réussite de ses projets. L'autorité de Korolev n'était bien sûr pas omnipotente : il devait composer avec ses collègues, voire céder face à eux. Prenons par exemple la façon d'envisager le déroulement du vol de Gagarin (1961). Korolev, parce qu'il pensait les cosmonautes comme un élément passif d'un système technique complexe, voulait limiter les gestes de Gagarin. Le responsable du corps des cosmonautes, Nikolaj Kamanin, les voyait, lui, comme des pilotes de l'espace. D'après lui, Gagarin devait réaliser des opérations de contrôle et rapporter au centre de contrôle les paramètres de vol. La position de Kamanin l'a finalement emportée : si le vol s'est déroulé en mode automatique, Gagarin a bien effectué les opérations de contrôle. 
Le deuxième fil concerne justement les relations entre l'homme et la machine. La propagande présentait les cosmonautes comme des modèles pour le «nouvel homme soviétique », un concept promu par le XXII ${ }^{e}$ Congrès du PCUS en 1961. Dans les faits, leur rôle était des plus restreints lors des vols, les ingénieurs soviétiques ne leur faisant pas confiance : non pas qu'ils les considérassent incapables d'accomplir les tâches, mais ils craignaient leur imprédictibilité. Aussi, si les vaisseaux Vostok disposaient des modes manuel et automatique de pilotage, seul ce dernier était envisagé : les cosmonautes ne devaient servir qu'à pallier les éventuelles déficiences de la machine. Cependant, pour cela, il fallait eux-mêmes qu'ils soient devenus des automates et c'était précisément l'objectif de l'entraînement, très dur, auxquels ils étaient soumis. Pourtant, comme le rappelle S. Gerovitch, la mission Voshod 2 (1965) aurait mal tourné si les cosmonautes n'avaient pas enfreint le règlement. Si l'emprise des machines a perduré avec la génération des véhicules Sojuz, elle a été remise en cause par la course à la lune. Au moins deux éléments appelaient en effet à une intervention humaine accrue : les limites de poids supposaient de réduire l'équipement embarqué et l'alunissage était une opération difficile.

Le troisième fil tient aux identités professionnelles des cosmonautes et des ingénieurs, dont chacune s'est construite dans leur relation mutuelle, parfois conflictuelle. Dans les premières années de l'aventure spatiale, les cosmonautes en sont les héros. Issus des rangs de l'aviation militaire, leur mission restait secrète jusqu'à son terme ; leurs proches eux-mêmes ignoraient la date du vol. Malgré leur formation et leurs compétences, ils devaient pourtant se contenter d'être soumis aux machines. En a résulté une profonde rancœur au sein du corps des cosmonautes qui a effectué un travail continu auprès des ingénieurs afin de modifier la division du travail entre l'homme et la machine. Une fois leur mission accomplie, et même si cela leur répugnait, les cosmonautes devenaient des personnages publics qui devaient avoir un comportement exemplaire. Mais les faire agir en hommes idéaux n'a pas toujours été sans difficulté. Les ingénieurs du programme spatial constituaient un groupe professionnel privilégié en Union soviétique. Longtemps resté dans l'ombre des cosmonautes, leur rôle n'a pas été mis en lumière avant la mort de Korolev (1966). D'après S. Gerovitch, leur identité était traversée par une tension profonde : le secret entourant leurs activités entretenait une affinité certaine avec les militaires, tandis que travailler sur des technologies de pointe nourrissait leur sentiment d'appartenance à l'élite internationale.

Le dernier fil a trait à la construction de la mythologie spatiale en Union soviétique et ses évolutions en Russie postsoviétique. L'ouvrage indique combien les voix qui l'ont forgée étaient nombreuses et signale les frictions qui ont résulté de cette multiplicité. Par exemple, la volonté de Kamanin de publiciser la vie des cosmonautes a embarrassé la hiérarchie militaire et les idéologues du Parti qui avaient l'impression de perdre la main sur la propagande. En effet, comme l'écrit S. Gerovitch, « le Parti communiste et l'État soviétique ont activement encouragé la création et la diffusion des mythes spatiaux, mais ces mythes n'ont pas simplement été imposés par en haut» (p. 26). Les mêmes protagonistes pouvaient 
aussi bien entretenir les mythes que fabriquer des « contre-mythes ». Dès lors, si les «mythes » publics servaient à incarner des concepts idéologiques abstraits et donner une cohésion au corps social, les « contre-mythes » renforçaient l'identité professionnelle des ingénieurs et des cosmonautes. Les organes de la propagande délivraient eux-mêmes des messages ambivalents et leur discours n'était pas d'une cohérence absolue. C'est ainsi le cas à propos des cosmonautes qu'ils dépeignaient à la fois comme des hommes extraordinaires honorant leur pays et comme des citoyens ordinaires symbolisant le peuple soviétique.

$\mathrm{Au}$ final, S. Gerovitch apporte une contribution majeure à l'étude des utopies scientifiques, du fonctionnement des mondes scientifiques et techniques, et des rôles attribués au progrès technique et aux machines dans le second $\mathrm{xx}^{\mathrm{e}}$ siècle. Les spécificités soviétiques apparaissent d'autant mieux que des comparaisons ponctuelles sont réalisées avec le programme spatial américain. Contrairement à l'Union soviétique où existait un éclatement institutionnel, les États-Unis avaient mis en place une agence spécifique : la NASA. C'est par exemple ce qui explique qu'on n'y retrouvait pas l'opposition professionnelle entre cosmonautes et ingénieurs. La lecture de ce livre passionnant pourra être complétée par celle d'un recueil de treize entretiens réalisés par $\mathrm{S}$. Gerovitch auprès de cosmonautes, d'ingénieurs et de militaires ${ }^{1}$.

1 - Slava Gerovitch, Voices of the Soviet Space Program : Cosmonauts, Soldiers, and Engineers Who Took the USSR into Space, New York : Palgrave MacMillan, 2014.

\section{Grégory Dufaud}

Sciences Po Lyon 